\section{NP32 Summer Harvest Adventure: A Garden- Based Obesity Prevention Program for Children Residing in Low-Resource Communities}

Colleen Spees, PhD, MEd, LD, RDN, FAND, spees.11@osu.edu, Ohio State University College of Medicine, 453 W 10th Avenue, 170 Atwell Hall, Columbus, OH 43210; Jenny Lobb, MPH, LD, RDN, Ohio State University Extension; James Portner, Ohio State University College of Social Work; Ashlea Braun, MS, LD, $R D N$, Ohio State University College of Medicine; Ingrid Adams, PhD, LD, RDN, Ohio State University Extension

Objective: The healthcare cost of every U.S. obese child over a lifetime is $\$ 19,000$ more than his/her normalweight counterpart. These financial costs are in addition to the devastating emotional, physical, and medical consequences of obesity. Childhood obesity remains a significant health issue, especially in disparate populations and during the summer months, when millions of children lose access to school-based feeding programs and suffer gaps in meals contributing to an obesogenic environment. The objective of this study is to test the efficacy of a summer comprehensive garden-based behavioral, social, and environmental intervention for children residing in low-resource communities.

Description: We will conduct a two-arm randomized controlled trial in low-resource children and parent/adult caregiver (PAC). A total of 240 children (ages 8-11 years) will be randomized to the garden-based intervention or an enhanced control group. The intervention consists of weekly group education, produce harvesting, remote motivational interviewing, and novel e-technologies. Assessments for both groups will be collected at orientation (week 0) and immediately following the completion of the intervention (week 10) by trained personnel that are blinded to participants' treatment arm assignment. Data on program satisfaction, quality of life, and family engagement will be collected via surveys. Anthropometrics, physical activity, and skin carotenoids will be measured objectively.

Evaluation: The current project will evaluate the efficacy of a garden-based intervention aimed at promoting modifiable lifestyle behaviors (diet and physical activity) and improving child-PAC interactions to promote an antiobesogenic environment for low-resource families during the summer months. We anticipate that compared to control, garden youths will exhibit greater improvements in fruit and vegetable intake; physical activity, quality of life, and indices of health; and child-PAC interactions. Conclusions and Implications: The information gleaned from this project will be used to inform, educate, and empower families and to establish a best practicebased summer obesity prevention model, built upon replicable criteria that can be implemented on a national scale.

Funding: 2017-68001-26353.

\section{NP33 Supermarket Science: Multipronged Approaches to Increasing Fresh, Frozen and Canned Fruit and Vegetable Purchases}

Michele Polacsek,PhD, MHS, mpolacsek@une.edu, University of New England, 716 Stevens Avenue, Portland, ME 04103; Alyssa Moran, PhD, MPH, RDN, Harvard T.H. Chan School of Public Health; Rebecca Boulos, PhD, MPH, University of Southern Maine; Anne Thorndike, MD, Harvard Medical School; Rebecca Franckle, ScD, Harvard T.H. Chan School of Public Health; Jason Block, MPH, MD, Harvard Medical School; Eric Rimm, ScD, Harvard T.H. Chan School of Public Health

Objective: This project aims to evaluate the success of a financial incentive combined with a point of purchase educational program to promote fruit and vegetable (FV) purchases and consumption in a supermarket setting among low-income and SNAP-eligible families and children living in a rural Maine community. Lessons learned from the research will inform the development of resources, training, professional practice and improved end user experience and behavior.

Description: The project uses a double value coupon incentive program combined with Cooking Matters at the Store education to incentivize the purchase and consumption of fresh produce, and healthful frozen and canned FV (without syrup or salt), nutrient-rich and affordable yearround alternatives to fresh produce. Education and extension efforts based on our study findings will contribute to meeting USDA Childhood Obesity Prevention Challenge Area Goals. The project team partnered with a large national retail grocery chain, Cooking Matters, University of Maine Cooperative Extension, eXtension Communities of Practice, Maine SNAP-Ed, and The Food Trust.

Evaluation: We used a randomized controlled study enrolling customers from a low-income rural Maine supermarket. A double value incentive for fresh, frozen or canned FV and opportunities to participate in Cooking Matters station-style education while shopping was provided to a randomly-selected intervention arm for 6 months after three months of baseline tracking. We used sales data to compare weekly FV sales pre to post intervention, and between intervention and control arms. FV consumption among participants and an index child was measured using a self-administered Food Frequency Questionnaire. All research, education and extension activities will be monitored.

Conclusions and Implications: The research phase of our project provides important information for policymakers interested in SNAP. Financial incentives combined with supermarket education has the potential to improve FV purchases and consumption. However, participation and scalability of the educational component remain a challenge.

Funding: 2016-68001-24961. 